\title{
A 3D Pose Estimator for the Visually Impaired
}

\author{
Joel A. Hesch, Faraz M. Mirzaei, Gian Luca Mariottini, and Stergios I. Roumeliotis \\ Dept. of Computer Science and Engineering, University of Minnesota \\ Email: $\{$ joel|faraz|gianluca|stergios $\} @$ cs.umn.edu
}

\begin{abstract}
This paper presents an indoor localization system for the visually impaired. The basis of our system is an Extended Kalman Filter (EKF) for six degree-of-freedom (d.o.f.) position and orientation (pose) estimation. The sensing platform consists of an Inertial Measurement Unit (IMU) and a 2D laser scanner. The IMU measurements are integrated to obtain pose estimates which are subsequently corrected using line-toplane correspondences between linear segments in the laserscan data and known 3D structural planes of the building. Furthermore, we utilize Lie derivatives to show that the system is observable when at least three planes are detected by the laser scanner. Experimental results are presented that demonstrate the reliability of the proposed method for accurate and realtime indoor localization.
\end{abstract}

\section{INTRODUCTION}

For a human, safe and efficient navigation requires obstacle avoidance, path planning, and determining one's position and orientation (pose) with respect to the world. For a visually impaired person, these tasks may be exceedingly difficult to accomplish, and there are high risks associated with failure in any of them. To address these issues, guide dogs and white canes are widely used for the purposes of wayfinding and environment sensing. The former, however, has costly and often prohibitive training requirements, while the latter can only provide cues about one's immediate surroundings. On the other hand, commercially available electronic navigation systems designed for the visually impaired (e.g., Humanware Trekker and Sendero BrailleNote ${ }^{1}$ ) rely on GPS signals, and cannot be utilized indoors, under tree cover, or next to tall buildings where reception is poor. Other existing navigation systems utilize body-mounted sensor packages which require the user to wear an electronic vest, or belt, fitted with sensing devices [1]. Unfortunately, these must be calibrated for each person's body, they often interfere with regular activities, and they may prevent a person from comfortably wearing certain articles of clothing (e.g., a jacket).

In contrast to these systems, we have recently presented an indoor localization aid utilizing a sensor package mounted on a white cane [2]. The main advantages of employing such a platform are: (i) the sensor package is unobtrusive to the user, (ii) there is no need to calibrate the system to specific body types, and (iii) the person maintains the ability

This work was supported by the University of Minnesota (DTC), and the National Science Foundation (IIS-0643680, IIS-0811946, IIS-0835637). J. A. Hesch was supported by the NIH Neuro-physical-computational Sciences Fellowship. F. M. Mirzaei was supported by the UMN Doctoral Dissertation Fellowship.

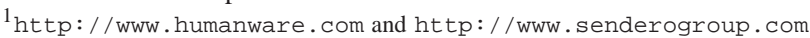

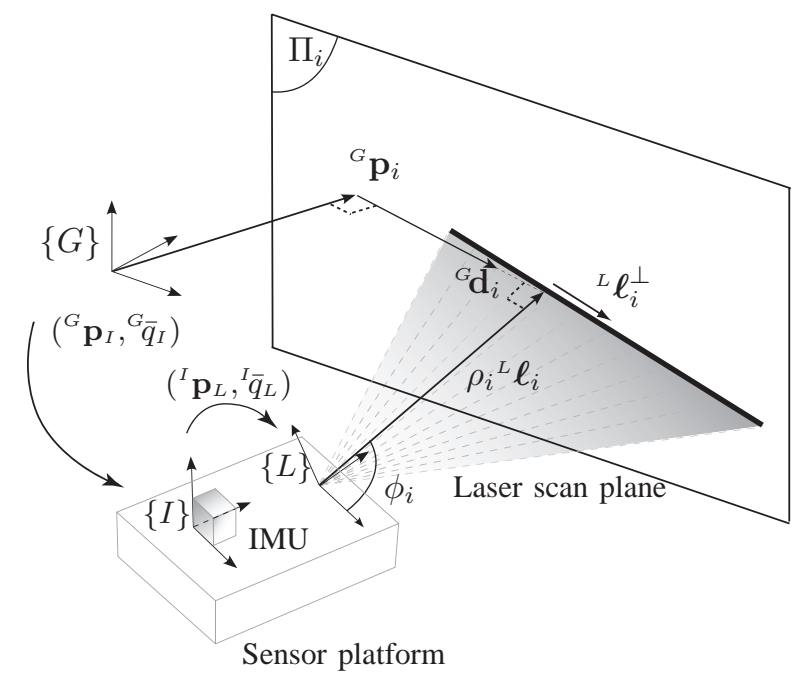

Fig. 1. As the IMU-laser sensor platform moves, the laser scan plane intersects a known planar surface, $\Pi_{i}$, described by ${ }^{G} \mathbf{p}_{i}$, with respect to the global frame of reference, $\{G\}$. The shortest vector in the laser scan plane from the origin of the laser frame, $\{L\}$, to $\Pi_{i}$ has length $\rho_{i}$ and direction ${ }^{L} \boldsymbol{\ell}_{i}$, with respect to $\{L\}$. The line of intersection has direction, ${ }^{L} \boldsymbol{\ell}_{i}^{\perp}$, with respect to $\{L\}$ and is described by the polar parameters $\left(\rho_{i}, \phi_{i}\right)$. The vector from the intersection of ${ }^{G} \mathbf{p}_{i}$ and $\Pi_{i}$ to the intersection of $\rho_{i}{ }^{L} \boldsymbol{\ell}_{i}$ and $\Pi_{i}$, is ${ }^{G} \mathbf{d}_{i}$. The known IMU-laser transformation is denoted by $\left({ }^{I} \mathbf{p}_{L},{ }^{I} \bar{q}_{L}\right)$, while the IMU pose with respect to $\{G\}$ is $\left({ }^{G} \mathbf{p}_{I},{ }^{G} \bar{q}_{I}\right)$.

to physically touch the environment with the white cane. The sensor package included a 3-axis gyroscope and a 2D laser scanner providing attitude information about the cane, as well as a foot-mounted pedometer measuring the person's walking speed. A two-layered estimator was employed to fuse the measurements and, by exploiting a known map of the environment, provided a $2.5 \mathrm{D}$ pose estimate of the person (i.e., 3 degree-of-freedom (d.o.f.) orientation and 2 d.o.f. position). Despite the novelty and success of the $2.5 \mathrm{D}$ system, it has certain limitations. First, estimating only 2 d.o.f. of the position prohibits the tracking of nonplanar motions (e.g., climbing stairs). Second, mounting the pedometer on the person's shoe, while the IMU and the laser scanner are mounted on the cane, results in an unknown, time-varying transformation between the sensors which prevents optimal fusion of their measurements.

To address these issues, in this work we replace the shoemounted pedometer and the gyroscopes with a cane-mounted Inertial Measurement Unit (IMU), which measures linear accelerations and rotational velocities (cf. Fig. 1). Additionally, we employ an Extended Kalman Filter (EKF) to integrate 
the IMU measurements and provide 6 d.o.f. pose estimates. However, without corrections, the IMU measurement noise and bias drift would cause the pose estimation errors to grow unbounded over time. To mitigate this issue, we update the pose estimates using straight-line features extracted from the 2D laser scans. In particular, as the person moves, the laser scanner's attitude changes which allows its scanning plane to intersect a variety of structural planes of the building (i.e., walls, floor, and ceiling), whose map is available a priori.

Furthermore, in order to initialize the EKF pose we extend Chen's algorithm [3] for line-to-plane pose determination so that it can utilize measurements from the laser scanner and the IMU. In addition, we present a study of the system observability based on Lie derivatives [4] which shows that the sensor measurements provide sufficient information for accurate pose estimation when three planes are observed. Experimental results are presented from a $120 \mathrm{~m}$ test run to validate the proposed method and quantify its accuracy.

The remainder of the paper is organized as follows. In Section II, we begin with an overview of the related literature. Section III presents the EKF-based pose estimator, while Section IV describes the initialization phase. The system observability is studied in Section V. The proposed method is validated with experimental results in Section VI, and we conclude the paper and present future directions in Section VII.

\section{RELATED WORK}

Recent work has focused primarily on developing hazarddetection aids for the visually impaired. These employ sensors for obstacle avoidance such as hand-held laser pointers [5], and sonars on a wheelchair [6], or on a robot connected at the tip of a white cane [7]. Cameras have also been used for object description (in terms of color and size) in addition to obstacle detection [8]. While these devices augment the perceptual abilities of a blind person and reduce the probability of an accident, they cannot be directly used as wayfinding aids without the development of appropriate algorithms for localization.

In contrast to the above systems, navigation aids have been designed that explicitly track a person's location and heading direction. Most relevant efforts have primarily addressed GPS-based outdoor navigation which cannot be used inside a building [9], [10]. Indoor navigation is more challenging, since pose information can only be inferred from the environment. Indoor navigation methods can be divided into three categories:

1) Dead-reckoning systems: These methods track a person's pose without any external reference. The most common approaches are based on foot-mounted accelerometers [11]. As a person walks, their position is computed by double integration of the acceleration measurements. Unfortunately, the integration of accelerometer bias and noise causes the position error to grow unbounded. Even if the rate of position-error increase can be reduced with static-period drift corrections [12], dead-reckoning systems still remain unreliable over long time intervals.
2) Beacon-based systems: Unlike dead-reckoning approaches that have no external references, these methods can infer position information by detecting uniquely identifiable beacons (or tags) installed in known locations throughout the building (e.g., by an elevator or bathroom). For example, in [13], a robot is attached at the end of a leash as a substitute for a guide dog, and localizes using odometry and a network of Radio Frequency IDentification (RFID) tags. Legge et al. presented another approach in which a hand-held camera identifies retro-reflective digital signs [14]. Similar methods also exist based on ultrasound [10] and infrared [15] beacons. In many cases the position estimates are only available when the person is in close proximity to a beacon and the person is left to navigate on their own during the inter-beacon periods. Another drawback of these approaches is the significant time and cost spent installing and calibrating beacons.

3) Feature-based systems: To mitigate the issues of beacon-based localization, commonly-occurring indoor features (e.g., doors, lamps or walls) can be exploited for localization with the help of appropriate sensors. Cameras are frequently used for this purpose as they can measure color, texture, and geometric shapes. Dellaert and Tariq [16], for example, proposed a multi-camera rig for 6 d.o.f. pose estimation for the visually impaired. Vision-based methods for pose estimation with portable fish-eye and omnidirectional cameras are described in [17] and [18], respectively. However, cameras require good lighting conditions, and the computational cost of high-dimensional feature extraction and processing is typically prohibitive for implementation on a portable device.

Unlike cameras, laser scanners can be utilized in a wide variety of lighting conditions and robustly detect low-level features (e.g., straight lines and corners) which can be efficiently matched. In the robotics community, 2D laser scanners have been employed for the purpose of $2 \mathrm{D}$ pose estimation (e.g., [19], [20]). Planar robot motion has also been tracked using a rotating $2 \mathrm{D}$ laser scanner (to emulate a $3 \mathrm{D}$ scan) [21]. In this case, the 3D information is also utilized to build a 3D representation of the world. While dense 3D laser scanning provides significantly more information for pose estimation, it is inappropriate for use on a navigation aid for the visually impaired due to weight limitations and computational cost.

For this reason, we have focus on 2D laser-based systems. We extend our previous work in [2], with a new navigation aid that estimates the 6 d.o.f. pose of a visually impaired person. We utilize an IMU and a 2D laser scanner along with a known map of structural planes in the building to track the person's pose. To the best of our knowledge, this is the first work on 3D pose tracking using a 2D laser scanner. The advantages compared to our previous work are: (i) tracking non-planar motions, thus allowing the visually impaired to traverse a wider variety of locations, (ii) the sensors are rigidly connected, which increases the accuracy of the pose estimate by removing the unknown, time-varying transformation from the system, (iii) we prove that the system is observable (i.e., we can accurately estimate the 
pose) when three walls are detected, and (iv) we provide an automated method to initialize the system using laser-scanner measurements.

\section{Localization Algorithm Description}

We hereafter address the problem of 6 d.o.f. pose estimation for localizing a visually-impaired person in an indoor environment. We assume that the position and orientation of the structural planes of the building are known from the blueprints. The visually-impaired person is equipped with an IMU and a 2D laser scanner, which are rigidly connected. The measurements from these two sensors are combined by an EKF estimator for tracking the pose of the person walking indoors. In the following section, we present the state and covariance propagation and update models used by the EKF, while the filter initialization is discussed in Section IV.

\section{A. Filter Propagation}

The EKF estimates the IMU pose and linear velocity together with the time-varying biases in the IMU signals. The filter state is the $16 \times 1$ vector:

$$
\mathbf{x}=\left[\begin{array}{lllll}
{ }^{I} \bar{q}_{G}^{T} & \mathbf{b}_{g}^{T} & { }^{G} \mathbf{v}_{I}^{T} & \mathbf{b}_{a}^{T} & { }^{G} \mathbf{p}_{I}^{T}
\end{array}\right]^{T},
$$

where ${ }^{I} \bar{q}_{G}(t)$ is the unit quaternion representing the orientation of the global frame $\{G\}$ in the IMU frame, $\{I\}$, at time $t$. The frame $\{G\}$ is an inertial reference frame affixed to the building, while $\{I\}$ is attached to the IMU (cf. Fig. 1). The position and velocity of $\{I\}$ in $\{G\}$ are denoted by the $3 \times 1$ vectors ${ }^{G} \mathbf{p}_{I}(t)$ and ${ }^{G} \mathbf{v}_{I}(t)$, respectively. The biases, $\mathbf{b}_{g}(t)$ and $\mathbf{b}_{a}(t)$, affecting the gyroscope and accelerometer measurements, are modeled as random walk processes driven by the zero-mean, white Gaussian noises $\mathbf{n}_{w g}(t)$ and $\mathbf{n}_{w a}(t)$, respectively.

1) Continuous-time model: The system model describing the time evolution of the state is (cf. [22], [23]):

$$
\begin{aligned}
{ }^{I} \dot{\bar{q}}_{G}(t) & =\frac{1}{2} \boldsymbol{\Omega}(\boldsymbol{\omega}(t))^{I} \bar{q}_{G}(t) \\
{ }^{G} \dot{\mathbf{p}}_{I}(t) & ={ }^{G} \mathbf{v}_{I}(t), \quad{ }^{G} \dot{\mathbf{v}}_{I}(t)={ }^{G} \mathbf{a}(t) \\
\dot{\mathbf{b}}_{g}(t) & =\mathbf{n}_{w g}(t), \quad \dot{\mathbf{b}}_{a}(t)=\mathbf{n}_{w a}(t) .
\end{aligned}
$$

In these expressions, $\boldsymbol{\omega}(t)=\left[\begin{array}{lll}\omega_{1}(t) & \omega_{2}(t) & \omega_{3}(t)\end{array}\right]^{T}$ is the rotational velocity of the IMU, expressed in $\{I\},{ }^{G} \mathbf{a}$ is the IMU acceleration expressed in $\{G\}$, and

$\boldsymbol{\Omega}(\boldsymbol{\omega})=\left[\begin{array}{cc}-\lfloor\boldsymbol{\omega} \times\rfloor & \boldsymbol{\omega} \\ -\boldsymbol{\omega}^{T} & 0\end{array}\right], \quad\lfloor\boldsymbol{\omega} \times\rfloor=\left[\begin{array}{ccc}0 & -\omega_{3} & \omega_{2} \\ \omega_{3} & 0 & -\omega_{1} \\ -\omega_{2} & \omega_{1} & 0\end{array}\right]$.

The gyroscope and accelerometer measurements, $\boldsymbol{\omega}_{m}$ and $\mathbf{a}_{m}$, used for state propagation, are:

$$
\begin{aligned}
\boldsymbol{\omega}_{m}(t) & =\boldsymbol{\omega}(t)+\mathbf{b}_{g}(t)+\mathbf{n}_{g}(t) \\
\mathbf{a}_{m}(t) & =\mathbf{C}\left({ }^{I} \bar{q}_{G}(t)\right)\left({ }^{G} \mathbf{a}(t)-{ }^{G} \mathbf{g}\right)+\mathbf{b}_{a}(t)+\mathbf{n}_{a}(t)
\end{aligned}
$$

where $\mathbf{n}_{g}$ and $\mathbf{n}_{a}$ are zero-mean, white Gaussian noise processes, and ${ }^{G} \mathbf{g}$ is the gravitational acceleration. The matrix $\mathbf{C}(\bar{q})$ is the rotation matrix corresponding to $\bar{q}$.

Linearizing about the current estimates and applying the expectation operator on both sides of (2)-(4), we obtain the state estimate propagation model

$$
\begin{aligned}
{ }^{I} \dot{\bar{q}}_{G}(t) & =\frac{1}{2} \boldsymbol{\Omega}(\hat{\boldsymbol{\omega}}(t))^{I} \hat{\bar{q}}_{G}(t) \\
{ }^{G} \dot{\hat{\mathbf{p}}}_{I}(t) & ={ }^{G} \hat{\mathbf{v}}_{I}(t), \quad{ }^{G} \dot{\hat{\mathbf{v}}}_{I}(t)=\mathbf{C}^{T}\left({ }^{I} \hat{\bar{q}}_{G}(t)\right) \hat{\mathbf{a}}(t)+{ }^{G} \mathbf{g} \\
\dot{\hat{\mathbf{b}}}_{g}(t) & =\mathbf{0}_{3 \times 1}, \quad \dot{\hat{\mathbf{b}}}_{a}(t)=\mathbf{0}_{3 \times 1}
\end{aligned}
$$

with

$$
\hat{\mathbf{a}}(t)=\mathbf{a}_{m}(t)-\hat{\mathbf{b}}_{a}(t) \text {, and } \hat{\boldsymbol{\omega}}(t)=\boldsymbol{\omega}_{m}(t)-\hat{\mathbf{b}}_{g}(t) .
$$

The $15 \times 1$ error-state vector is defined as:

$$
\widetilde{\mathbf{x}}=\left[\begin{array}{llllll}
{ }^{I} \boldsymbol{\delta} \boldsymbol{\theta}_{G}^{T} & \widetilde{\mathbf{b}}_{g}^{T} & { }^{G} \widetilde{\mathbf{v}}_{I}^{T} & \widetilde{\mathbf{b}}_{a}^{T} & { }^{G} \widetilde{\mathbf{p}}_{I}^{T}
\end{array}\right]^{T} .
$$

For the IMU position, velocity, and biases, an additive error model is utilized (i.e., the error in the estimate $\hat{x}$ of a quantity $x$ is $\widetilde{x}=x-\hat{x}$ ). However, for the quaternion a different error definition is employed. In particular, if $\hat{\bar{q}}$ is the estimated value of the quaternion $\bar{q}$, then the attitude error $\boldsymbol{\delta} \boldsymbol{\theta}$ is implicitly defined by the error quaternion:

$$
\delta \bar{q}=\bar{q} \otimes \hat{\bar{q}}^{-1} \simeq\left[\begin{array}{ll}
\frac{1}{2} \boldsymbol{\delta} \boldsymbol{\theta}^{T} & 1
\end{array}\right]^{T}
$$

where $\delta \bar{q}$ describes the (small) rotation that causes the true and estimated attitude to coincide. The main advantage of this error definition is that it allows us to represent the attitude uncertainty by the $3 \times 3$ covariance matrix $E\left\{\boldsymbol{\delta} \boldsymbol{\theta} \boldsymbol{\delta} \boldsymbol{\theta}^{T}\right\}$. Since the attitude corresponds to 3 d.o.f., this is a minimal representation.

The linearized continuous-time error-state equation is:

$$
\dot{\tilde{\mathbf{x}}}=\mathbf{F}_{c} \widetilde{\mathbf{x}}+\mathbf{G}_{c} \mathbf{n},
$$

where

$$
\begin{array}{r}
\mathbf{F}_{c}=\left[\begin{array}{ccccc}
-\lfloor\hat{\boldsymbol{\omega}} \times\rfloor & -\mathbf{I}_{3} & \mathbf{0}_{3 \times 3} & \mathbf{0}_{3 \times 3} & \mathbf{0}_{3 \times 3} \\
\mathbf{0}_{3 \times 3} & \mathbf{0}_{3 \times 3} & \mathbf{0}_{3 \times 3} & \mathbf{0}_{3 \times 3} & \mathbf{0}_{3 \times 3} \\
-\mathbf{C}^{T}\left({ }^{I} \hat{\bar{q}}_{G}\right)\lfloor\hat{\mathbf{a}} \times\rfloor & \mathbf{0}_{3 \times 3} & \mathbf{0}_{3 \times 3} & -\mathbf{C}^{T}\left({ }^{I} \hat{\bar{q}}_{G}\right) & \mathbf{0}_{3 \times 3} \\
\mathbf{0}_{3 \times 3} & \mathbf{0}_{3 \times 3} & \mathbf{0}_{3 \times 3} & \mathbf{0}_{3 \times 3} & \mathbf{0}_{3 \times 3} \\
\mathbf{0}_{3 \times 3} & \mathbf{0}_{3 \times 3} & \mathbf{I}_{3} & \mathbf{0}_{3 \times 3} & \mathbf{0}_{3 \times 3}
\end{array}\right] \\
\mathbf{G}_{c}=\left[\begin{array}{cccc}
-\mathbf{I}_{3} & \mathbf{0}_{3 \times 3} & \mathbf{0}_{3 \times 3} & \mathbf{0}_{3 \times 3} \\
\mathbf{0}_{3 \times 3} & \mathbf{I}_{3} & \mathbf{0}_{3 \times 3} & \mathbf{0}_{3 \times 3} \\
\mathbf{0}_{3 \times 3} & \mathbf{0}_{3 \times 3} & -\mathbf{C}^{T}\left({ }^{I} \bar{q}_{G}\right) & \mathbf{0}_{3 \times 3} \\
\mathbf{0}_{3 \times 3} & \mathbf{0}_{3 \times 3} & \mathbf{0}_{3 \times 3} & \mathbf{I}_{3} \\
\mathbf{0}_{3 \times 3} & \mathbf{I}_{3} & \mathbf{0}_{3 \times 3} & \mathbf{0}_{3 \times 3}
\end{array}\right] \quad \mathbf{n}=\left[\begin{array}{c}
\mathbf{n}_{g} \\
\mathbf{n}_{w g} \\
\mathbf{n}_{a} \\
\mathbf{n}_{w a}
\end{array}\right]
\end{array}
$$

where $\mathbf{I}_{3}$ is the $3 \times 3$ identity matrix. The system noise covariance matrix $\mathbf{Q}_{c}$ depends on the IMU noise characteristics and is computed off-line [23].

2) Discrete-time implementation: The IMU signals $\boldsymbol{\omega}_{m}$ and $\mathbf{a}_{m}$ are sampled at a constant rate $1 / T$. Every time a new IMU measurement is received, the state estimate is propagated using 4th-order Runge-Kutta numerical integration of (7)-(9). In order to derive the covariance propagation equation, we evaluate the discrete-time state transition matrix:

$$
\boldsymbol{\Phi}_{k}=\boldsymbol{\Phi}\left(t_{k+1}, t_{k}\right)=\exp \left(\int_{t_{k}}^{t_{k+1}} \mathbf{F}_{c}(\tau) \mathrm{d} \tau\right)
$$


and the discrete-time system noise covariance matrix:

$$
\mathbf{Q}_{d, k}=\int_{t_{k}}^{t_{k+1}} \boldsymbol{\Phi}\left(t_{k+1}, \tau\right) \mathbf{G}_{c} \mathbf{Q}_{c} \mathbf{G}_{c}^{T} \boldsymbol{\Phi}^{T}\left(t_{k+1}, \tau\right) \mathrm{d} \tau .
$$

The propagated covariance is then computed as:

$$
\mathbf{P}_{k+1 \mid k}=\boldsymbol{\Phi}_{k} \mathbf{P}_{k \mid k} \boldsymbol{\Phi}_{k}^{T}+\mathbf{Q}_{d, k}
$$

\section{B. Filter Update}

As the IMU-laser platform moves in an indoor environment, the laser-scan plane intersects the known structural planes, $\Pi_{i}$, along line segments with direction ${ }^{L} \ell_{i}^{\perp}$ (cf. Fig. 1). Each line is uniquely described in the laser frame, $\{L\}$, by $\left(\rho_{i}, \phi_{i}\right)$, where $\rho_{i}$ is the distance from the origin of $\{L\}$ to the line, and $\phi_{i}$ is the angle of the vector ${ }^{L} \boldsymbol{\ell}_{i}$ perpendicular to the line ${ }^{2}$. We will hereafter express the line direction in $\{I\}$, as ${ }^{I} \ell_{i}^{\perp}=\mathbf{C}\left({ }^{I} \bar{q}_{L}\right)\left[\sin \phi_{i}-\cos \phi_{i} 0\right]^{T}$, where ${ }^{I} \bar{q}_{L}$ is the unit quaternion representing the orientation of the laser frame in the IMU frame ${ }^{3}$. In what follows, we describe how each line is exploited to define two measurement constraints, used by the EKF to update the state estimates.

1) Orientation Constraint: The first constraint is on the orientation of $\{I\}$ with respect to $\Pi_{i}$. Each plane is uniquely described by ${ }^{G} \mathbf{p}_{i}$, which is the shortest vector from the origin of $\{G\}$ to $\Pi_{i}$ and is known from the blueprints. The vector ${ }^{G} \mathbf{p}_{i}$ is perpendicular to $\mathbf{C}^{T}\left({ }^{I} \bar{q}_{G}\right){ }^{I} \boldsymbol{\ell}_{i}^{\perp}$ (cf. Fig. 1), which yields the following orientation measurement constraint:

$$
z_{1, i}={ }^{G} \mathbf{p}_{i}^{T} \mathbf{C}^{T}\left({ }^{I} \bar{q}_{G}\right){ }^{I} \boldsymbol{\ell}_{i}^{\perp}=0 .
$$

The expected measurement is

$$
\hat{z}_{1, i}={ }^{G} \mathbf{p}_{i}^{T} \mathbf{C}^{T}\left({ }^{I} \hat{\bar{q}}_{G}\right){ }^{I} \boldsymbol{\ell}_{m i}^{\perp},
$$

where ${ }^{I} \boldsymbol{\ell}_{m i}^{\perp}=\mathbf{C}\left({ }^{I} \bar{q}_{L}\right)\left[\sin \phi_{m i}-\cos \phi_{m i} 0\right]^{T}$ is the measured line direction with $\phi_{m i}=\phi_{i}-\tilde{\phi}_{i}$. The measurement residual is $r_{1, i}=z_{1, i}-\hat{z}_{1, i}=-\hat{z}_{1, i}$ and the corresponding linearized error model is

$$
\begin{aligned}
& \widetilde{z}_{1, i} \simeq\left[\begin{array}{ll}
-{ }^{G} \mathbf{p}_{i}^{T} \mathbf{C}^{T}\left({ }^{I} \hat{\bar{q}}_{G}\right)\left\lfloor{ }^{I} \boldsymbol{\ell}_{m i}^{\perp} \times\right\rfloor & \mathbf{0}_{1 \times 12}
\end{array}\right] \widetilde{\mathbf{x}}+ \\
& {\left[{ }^{G} \mathbf{p}_{i}^{T} \mathbf{C}^{T}\left({ }^{I} \hat{\bar{q}}_{G}\right)^{I} \boldsymbol{\ell}_{m i} \quad 0\right]\left[\begin{array}{c}
\tilde{\phi}_{i} \\
\tilde{\rho}_{i}
\end{array}\right]} \\
& =\mathbf{h}_{1, i}^{T} \widetilde{\mathbf{x}}+\gamma_{1, i}^{T} \mathbf{n}_{\ell i}
\end{aligned}
$$

where ${ }^{I} \boldsymbol{\ell}_{m i}=\mathbf{C}\left({ }^{I} \bar{q}_{L}\right)\left[\begin{array}{lll}\cos \phi_{m i} & \sin \phi_{m i} & 0\end{array}\right]^{T}$ is the perpendicular to the measured line direction and $\rho_{m i}=\rho_{i}-\tilde{\rho}_{i}$ is the measured distance to the line.

The vectors $\mathbf{h}_{1, i}^{T}$ and $\gamma_{1, i}^{T}$ are the Jacobians of (17) with respect to the state and line fitting parameters, respectively. The error vector $\mathbf{n}_{\ell i}$ is assumed to be zero-mean, white Gaussian, with covariance matrix $\mathbf{R}_{i}=E\left\{\mathbf{n}_{\ell i} \mathbf{n}_{\ell i}^{T}\right\}$ computed from the weighted line-fitting procedure [25].

\footnotetext{
${ }^{2}$ We utilized the Split-and-Merge algorithm [24] to segment the laserscan data and a weighted line fitting algorithm [25] to estimate the line parameters $\left(\rho_{i}, \phi_{i}\right)$ for each line.

${ }^{3}$ The laser-to-IMU rigid transformation is assumed known and given by $\left({ }^{I} \mathbf{p}_{L},{ }^{I} \bar{q}_{L}\right)$. This can be obtained, for example, through a calibration procedure adapted from [26].
}

2) Distance Constraint: From Fig. 1, the following geometric relationship holds:

$$
{ }^{G} \mathbf{p}_{I}+\mathbf{C}^{T}\left({ }^{I} \bar{q}_{G}\right)\left({ }^{I} \mathbf{p}_{L}+\rho_{i}{ }^{I} \ell_{i}\right)={ }^{G} \mathbf{p}_{i}+{ }^{G} \mathbf{d}_{i}
$$

where ${ }^{I} \ell_{i}=\mathbf{C}\left({ }^{I} \bar{q}_{L}\right)\left[\cos \phi_{i} \sin \phi_{i} 0\right]^{T}$ is the perpendicular to the line direction. The vector ${ }^{G} \mathbf{d}_{i}$ is eliminated by projecting (20) onto ${ }^{G} \mathbf{p}_{i}^{T}$, yielding the distance measurement constraint

$$
z_{2, i}={ }^{G} \mathbf{p}_{i}^{T}\left({ }^{G} \mathbf{p}_{I}+\mathbf{C}^{T}\left({ }^{I} \bar{q}_{G}\right)\left({ }^{I} \mathbf{p}_{L}+\rho_{i}{ }^{I} \boldsymbol{\ell}_{i}\right)\right)-{ }^{G} \mathbf{p}_{i}^{T G} \mathbf{p}_{i}=0 .
$$

The expected measurement is

$$
\hat{z}_{2, i}={ }^{G} \mathbf{p}_{i}^{T}\left({ }^{G} \hat{\mathbf{p}}_{I}+\mathbf{C}^{T}\left({ }^{I} \hat{\bar{q}}_{G}\right)\left({ }^{I} \mathbf{p}_{L}+\rho_{m i}{ }^{I} \boldsymbol{\ell}_{m i}\right)\right)-{ }^{G} \mathbf{p}_{i}^{T G} \mathbf{p}_{i} .
$$

The measurement residual is $r_{2, i}=z_{2, i}-\hat{z}_{2, i}=-\hat{z}_{2, i}$ and the corresponding linearized error model is

$$
\begin{aligned}
\widetilde{z}_{2, i} \simeq & {\left[\begin{array}{lll}
-{ }^{G} \mathbf{p}_{i}^{T} \mathbf{C}^{T}\left({ }^{I} \hat{\bar{q}}_{G}\right)\left\lfloor{ }^{I} \mathbf{p}_{L}+\rho_{m i}{ }^{I} \boldsymbol{\ell}_{m i} \times\right\rfloor & \mathbf{0}_{1 \times 9} & { }^{G} \mathbf{p}_{i}^{T}
\end{array}\right] \widetilde{\mathbf{x}} } \\
& +\left[-\left[\begin{array}{ll}
-{ }^{G} \mathbf{p}_{i}^{T} \mathbf{C}^{T}\left({ }^{I} \hat{\bar{q}}_{G}\right) \rho_{m i}{ }^{I} \ell_{m i}^{\perp} & { }^{G} \mathbf{p}_{i}^{T} \mathbf{C}^{T}\left({ }^{I} \hat{\bar{q}}_{G}\right){ }^{I} \boldsymbol{\ell}_{m i}
\end{array}\right]\left[\begin{array}{c}
\tilde{\phi}_{i} \\
\tilde{\rho}_{i}
\end{array}\right]\right. \\
= & \mathbf{h}_{2, i}^{T} \widetilde{\mathbf{x}}+\gamma_{2, i}^{T} \mathbf{n}_{\ell i}
\end{aligned}
$$

where the vectors $\mathbf{h}_{2, i}^{T}$ and $\gamma_{2, i}^{T}$ are the Jacobians of (21) with respect to the state and line fitting parameters, respectively.

We process the two measurement constraints together; stacking (19) and (22) we obtain the measurement Jacobians $\mathbf{H}_{i}=\left[\begin{array}{ll}\mathbf{h}_{1, i} & \mathbf{h}_{2, i}\end{array}\right]^{T}$, and $\boldsymbol{\Gamma}_{i}=\left[\begin{array}{ll}\boldsymbol{\gamma}_{1, i} & \boldsymbol{\gamma}_{2, i}\end{array}\right]^{T}$ used in the expression for the Kalman gain

$$
\mathbf{K}_{i}=\mathbf{P}_{k+1 \mid k} \mathbf{H}_{i}^{T}\left(\mathbf{H}_{i} \mathbf{P}_{k+1 \mid k} \mathbf{H}_{i}^{T}+\boldsymbol{\Gamma}_{i} \mathbf{R}_{i} \boldsymbol{\Gamma}_{i}^{T}\right)^{-1} .
$$

The residual vector is $\mathbf{r}_{i}=\left[\begin{array}{ll}r_{1, i} & r_{2, i}\end{array}\right]^{T}$, and the state and the covariance update equations are given by

$$
\begin{aligned}
& \hat{\mathbf{x}}_{k+1 \mid k+1}=\hat{\mathbf{x}}_{k+1 \mid k}+\mathbf{K}_{i} \mathbf{r}_{i} \\
\mathbf{P}_{k+1 \mid k+1}= & \left(\mathbf{I}_{15}-\mathbf{K}_{i} \mathbf{H}_{i}\right) \mathbf{P}_{k+1 \mid k}\left(\mathbf{I}_{15}-\mathbf{K}_{i} \mathbf{H}_{i}\right)^{T}+ \\
& \mathbf{K}_{i} \boldsymbol{\Gamma}_{i} \mathbf{R}_{i} \boldsymbol{\Gamma}_{i}^{T} \mathbf{K}_{i}^{T} .
\end{aligned}
$$

\section{Zero-Velocity Update}

When the laser scanner does not detect any structural planes along certain directions for an extended period of time, the position estimates accumulate errors along those directions, due to accelerometer drift. This effect is closely related to the system's observability (Section V) and can be compensated by means of drift correction during instantaneous stationary periods of the motion [12].

This procedure, termed zero-velocity update, is challenging for two reasons: (i) the stationary periods must be identified without an external reference, and (ii) the IMU drift error must be corrected without violating the statistics of the pose estimate. Existing methods typically detect stationary periods based on a threshold check for the accelerometer measurement. These require significant hand tuning, and cannot account for the uncertainty in the current state estimate.

In contrast, we formulate the zero-velocity constraint as an EKF measurement and use the Mahalanobis distance test to identify the stationary intervals. Specifically, for the zero- 
velocity update, we employ the following measurement constraints for the linear acceleration, and linear and rotational velocities which are (instantaneously) equal to zero

$$
\mathbf{z}_{\zeta}=\left[\begin{array}{lll}
\mathbf{a}^{T} & \boldsymbol{\omega}^{T} & { }^{G} \mathbf{v}_{I}^{T}
\end{array}\right]^{T}=\mathbf{0}_{9 \times 1} .
$$

The zero-velocity measurement residual is

$$
\mathbf{r}_{\zeta}=\mathbf{z}_{\zeta}-\hat{\mathbf{z}}_{\zeta}=\left[\begin{array}{c}
-\mathbf{a}_{m}+\hat{\mathbf{b}}_{a}-\mathbf{C}\left({ }^{I} \hat{\bar{q}}_{G}\right)^{G} \mathbf{g} \\
-\boldsymbol{\omega}_{m}+\hat{\mathbf{b}}_{g} \\
-{ }^{G} \hat{\mathbf{v}}_{I}
\end{array}\right]
$$

and the corresponding linearized error model is

$$
\begin{aligned}
\widetilde{\mathbf{z}}_{\zeta} & \simeq\left[\begin{array}{ccccc}
-\left\lfloor\mathbf{C}\left({ }^{I} \hat{\bar{q}}_{G}\right)^{G} \mathbf{g} \times\right\rfloor & \mathbf{0}_{3 \times 3} & \mathbf{0}_{3 \times 3} & \mathbf{I}_{3} & \mathbf{0}_{3 \times 3} \\
\mathbf{0}_{3 \times 3} & \mathbf{I}_{3} & \mathbf{0}_{3 \times 3} & \mathbf{0}_{3 \times 3} & \mathbf{0}_{3 \times 3} \\
\mathbf{0}_{3 \times 3} & \mathbf{0}_{3 \times 3} & \mathbf{I}_{3} & \mathbf{0}_{3 \times 3} & \mathbf{0}_{3 \times 3}
\end{array}\right] \widetilde{\mathbf{x}}+\left[\begin{array}{c}
\mathbf{n}_{a} \\
\mathbf{n}_{g} \\
\mathbf{n}_{v}
\end{array}\right] \\
& =\mathbf{H}_{\zeta} \widetilde{\mathbf{x}}+\mathbf{n}_{\zeta},
\end{aligned}
$$

where $\mathbf{H}_{\zeta}$ is the Jacobian of the zero-velocity measurement with respect to the state, and $\mathbf{n}_{v}$ is a zero-mean, white Gaussian process noise that acts as a regularization term for computing the inverse of the measurement residuals' covariance. Based on this update model, at time step $k$ we compute the Mahalanobis distance $\chi^{2}=\mathbf{r}_{\zeta}^{T} \mathbf{S}_{k}^{-1} \mathbf{r}_{\zeta}$, where $\mathbf{S}_{k}=\mathbf{H}_{\zeta} \mathbf{P}_{k \mid k} \mathbf{H}_{\zeta}^{T}+\mathbf{R}_{\zeta}$ is the covariance of the measurement residual and $\mathbf{R}_{\zeta}=E\left\{\mathbf{n}_{\zeta} \mathbf{n}_{\zeta}^{T}\right\}$ is the measurement noise covariance. If $\chi^{2}$ is less than a chosen probabilistic threshold, a stationary interval is detected and the state vector and the covariance matrix are updated using (24)-(26). We note that once we use the inertial measurements for an update, we cannot use them for propagation. However, this is not an issue, since the IMU is static and we do not need to use the kinematic model (2)-(4) to propagate the state estimates. Instead we employ the following equations:

$$
\begin{gathered}
{ }^{I} \dot{\bar{q}}_{G}(t)=\mathbf{0}_{4 \times 1} \quad, \quad{ }^{G} \dot{\mathbf{p}}_{I}(t)=\mathbf{0}_{3 \times 1}, \quad{ }^{G} \dot{\mathbf{v}}_{I}(t)=\mathbf{0}_{3 \times 1} \\
\dot{\mathbf{b}}_{g}(t)=\mathbf{n}_{w g}(t), \quad \dot{\mathbf{b}}_{a}(t)=\mathbf{n}_{w a}(t) .
\end{gathered}
$$

In essence, this static-IMU propagation model indicates that the state vector and the covariance matrix of all components are kept constant. The only exceptions are the covariances of the errors in the gyroscope and accelerometer bias estimates which increase at each time step to reflect the effect of the random walk model.

\section{FiLter InItialization}

Before using the EKF to fuse measurements from the laser scanner and the IMU, we need to initialize the state vector estimate $\widehat{\mathbf{x}}_{0 \mid 0}$ along with its covariance $\mathbf{P}_{0 \mid 0}$. This is performed in four sequential stages: (i) the gyroscopes' biases $\mathbf{b}_{g}$ are initialized using the partial zero-velocity updates (Section IV-A), (ii) the IMU orientation ${ }^{I} \bar{q}_{G}$ is initialized employing the laser scans (Section IV-B), (iii) the accelerometers' biases $\mathbf{b}_{a}$ are initialized using zero-velocity updates (Section IV-C), and (iv) the IMU position ${ }^{G} \mathbf{p}_{I}$ is initialized employing the laser scans (Section IV-D).

\section{A. Gyroscopes' Biases Initialization}

The complete zero-velocity update described in Section III-C cannot be directly applied to initialize the gy- roscope biases. This is due to the fact that an estimate of the orientation ${ }^{I} \bar{q}_{G}$, required for evaluating $\mathbf{H}_{\zeta}$ [cf. (26)], cannot be obtained before estimating the gyroscope biases (Section IV-B.2). Instead, to provide an initial estimate for the gyroscope biases, $\mathbf{b}_{g}$, we use partial zero-velocity updates. In particular, we initially set $\hat{\mathbf{b}}_{g}$ to an arbitrary value (e.g., zero), while its covariance is set to a large value, reflecting the lack of a priori knowledge about the estimates. Then, we keep the IMU static (i.e., $\boldsymbol{\omega}=\mathbf{0}_{3 \times 1}$ ) and use the second components of (24)-(26) to perform a partial zerovelocity update.

\section{B. Orientation Initialization}

Since the IMU and the laser scanner are rigidly connected and their relative transformation is known, the initial orientation of the IMU can be directly computed from the initial orientation of the laser scanner. We describe two methods to compute the orientation of the laser scanner using line measurements of three planes with linearly-independent normal vectors. The first method, adapted from Chen [3], requires observation of all three planes from the same viewpoint, while the second method is capable of using laser scan measurements taken from different perspectives by exploiting the motion information from the gyroscopes.

1) Concurrent observation of three planes: When three non-parallel planes are scanned from the same viewpoint (i.e., the same frame of reference), the estimate of the orientation ${ }^{I} \bar{q}_{G}$ is initialized using Chen's method [3]. In this case, three quadratic constraints in terms of the unit quaternion ${ }^{I} \bar{q}_{G}$ are obtained from the laser scans [cf. (17)], each of them describing the relationship between a line measurement and the corresponding plane:

$$
z_{1, i}={ }^{G} \mathbf{p}_{i}^{T} \mathbf{C}^{T}\left({ }^{I} \bar{q}_{G}\right){ }^{I} \ell_{i}^{\perp}=0, \quad i=1, \ldots, 3 .
$$

Chen's algorithm employs the properties of rotation matrices in algebraic manipulations to convert this system of equations to an eighth-order univariate polynomial in one of the d.o.f. of the unknown rotation. Eight solutions for this univariate polynomial are obtained, for example, using the Companion matrix. The remaining two d.o.f. of the rotation ${ }^{I} \bar{q}_{G}$ are subsequently determined by back-substitution. In general, an external reference is required to distinguish the true solution among the eight possible ones. In our work, we employ the gravity measurement from the accelerometers and the planes' identities to find the unique solution.

2) Motion-aided orientation initialization: In order to use Chen's method for initializing the orientation, all three line measurements must be expressed with respect to the same frame of reference; hence three non-parallel planes must be concurrently observed by the laser scanner from the same viewpoint. However, satisfying this prerequisite is quite limiting since it requires facing a corner of a room, for example, where three structural planes intersect. In this work, we address this issue by using the gyroscope measurements to transform the laser scans taken from different viewpoints at different time instants to a common frame of reference. We choose as the common frame, the IMU frame when the 
first laser scan is recorded (i.e., at time $t_{1}$ ), and denote it by $\left\{I_{1}\right\}$. In this way, we can rewrite the inferred measurement constraints (17) at time $t_{j}, j=2,3$ as

$$
{ }^{G} \mathbf{p}_{j}^{T} \mathbf{C}^{T}\left({ }^{I} \bar{q}_{G}\left(t_{j}\right)\right)^{I} \boldsymbol{\ell}_{j}^{\perp}\left(t_{j}\right)={ }^{G} \mathbf{p}_{j}^{T} \mathbf{C}^{T}\left({ }^{I} \bar{q}_{G}\left(t_{1}\right)\right)^{I_{1}} \boldsymbol{\ell}_{j}^{\perp}\left(t_{j}\right)=0
$$

where ${ }^{I_{1}} \boldsymbol{\ell}_{j}^{\perp}\left(t_{j}\right)=\mathbf{C}\left({ }^{I_{1}} \bar{q}_{I_{j}}\right)^{I} \boldsymbol{\ell}_{j}^{\perp}\left(t_{j}\right)$ is the line direction corresponding to the plane $\Pi_{j}$, recorded at time $t_{j}$, and transformed to the frame $\left\{I_{1}\right\}$. Since the gyroscope biases are already initialized, the quaternions ${ }^{I_{1}} \bar{q}_{I_{j}}$ can be obtained by integrating the rotational velocity measurements [cf. (7) and (10)] between time instants $t_{1}$ and $t_{j}$. Once all the line directions ${ }^{I} \ell_{j}^{\perp}\left(t_{j}\right)$ are expressed with respect to $\left\{I_{1}\right\}$, we employ Chen's algorithm, described before, to find the initial orientation ${ }^{I} \bar{q}_{G}\left(t_{1}\right)$.

The covariance of the initial orientation estimate is obtained by computing the corresponding Jacobians [by linearizing (28)] and using the uncertainty (covariance) in the estimates of ${ }^{I} \ell_{j}^{\perp}$ and ${ }^{{ }^{I} 1} \bar{q}_{I_{j}}$. However, note that the estimates of the relative transformations ${ }^{I_{1}} \bar{q}_{I_{2}}$ and ${ }^{I_{1}} \bar{q}_{I_{3}}$ are correlated. To account for these correlations, we employ the stochastic cloning technique [27] to augment the state vector and the covariance matrix of the EKF with ${ }^{I_{1}} \bar{q}_{I_{j}}$ at time $t_{j}$ (assuming we have started integrating from time $t_{1}$ ). In this way, we are able to estimate the IMU orientation by integrating the gyroscope measurements, and concurrently compute the correlations between the IMU orientation estimates at the time instants when laser scans are recorded.

\section{Accelerometers' Biases Initialization}

In this step, similar to the gyroscope bias initialization, we set the estimate for the accelerometer biases, $\mathbf{b}_{a}$, to an arbitrary value (e.g., zero), and set its covariance to a sufficiently large value, representing our uncertainty about the arbitrary initial estimate. Since the IMU is initially static, we set the velocity estimate ${ }^{G} \mathbf{v}_{I}$ and its covariance to zero. Then, keeping the IMU static, we utilize the complete zerovelocity update described in Section III-C to initialize the accelerometer biases.

\section{Position Initialization}

In order to compute the initial position ${ }^{G} \mathbf{p}_{I}$, once the orientation ${ }^{I} \bar{q}_{G}$ is known, we rewrite the distance constraints (21) as $(j=1, \ldots, 3)$

$$
{ }^{G} \mathbf{p}_{j}^{T{ }^{G}} \mathbf{p}_{I}={ }^{G} \mathbf{p}_{j}^{T{ }^{G}} \mathbf{p}_{j}-{ }^{G} \mathbf{p}_{j}^{T} \mathbf{C}^{T}\left({ }^{I} \bar{q}_{G}\right)\left({ }^{I} \mathbf{p}_{L}+\rho_{j}{ }^{I} \ell_{j}\right) .
$$

Clearly, if ${ }^{G} \mathbf{p}_{j}, j=1, \ldots, 3$ are linearly independent, we can uniquely determine ${ }^{G} \mathbf{p}_{I}$ from these three equations. However, as mentioned before, concurrently observing three planes whose normal vectors are linearly independent is difficult in practice. To mitigate this issue, we employ an approach similar to the orientation initialization and use the IMU measurements to link the pose of the IMU when taking laser scans from different viewpoints (and thus at different time instants), and transform them to a common frame of reference. If we choose $\left\{I_{1}\right\}$, the IMU frame when recording the first laser scan, as the common frame of reference, we can express the distance constraints imposed by the laser scans at subsequent IMU poses, $\left\{I_{j}\right\}$, as $(j=2,3)$

$$
\begin{aligned}
{ }^{G} \mathbf{p}_{j}^{T{ }^{G}} \mathbf{p}_{I}= & { }^{G} \mathbf{p}_{j}^{T G} \mathbf{p}_{j}-{ }^{G} \mathbf{p}_{j}^{T} \mathbf{C}^{T}\left({ }^{I} \bar{q}_{G}\left(t_{1}\right)\right)^{I_{1}} \mathbf{p}_{I_{j}} \\
& -{ }^{G} \mathbf{p}_{j}^{T} \mathbf{C}^{T}\left({ }^{I} \bar{q}_{G}\left(t_{1}\right)\right) \mathbf{C}\left({ }^{I_{1}} \bar{q}_{I_{j}}\right)\left({ }^{I} \mathbf{p}_{L}+\rho_{j}\left(t_{j}\right)^{I} \boldsymbol{\ell}_{j}\left(t_{j}\right)\right)
\end{aligned}
$$

where ${ }^{I_{1}} \bar{q}_{I_{j}}$ and ${ }^{I_{1}} \mathbf{p}_{I_{j}}$ specify the transformation between $\left\{I_{1}\right\}$ and $\left\{I_{j}\right\}$ computed by integrating rotational velocities and linear accelerations, respectively [cf. (7)-(10)]. Notice that except ${ }^{G} \mathbf{p}_{I}$, all the quantities in (30) are known (either measured or estimated). Therefore, the two equations (30) as well as the first distance constraint at $\left\{I_{1}\right\}$ (i.e., (29) for $j=1$ ) are readily used to obtain ${ }^{G} \mathbf{p}_{I}$ assuming ${ }^{G} \mathbf{p}_{j}, j=$ $1, \ldots, 3$ are linearly independent.

Finally, we use the appropriate Jacobians [by linearizing (30)] along with the covariances of the estimates of the IMU pose $\left({ }^{{ }^{I}} \bar{q}_{I_{j}},{ }^{I_{1}} \mathbf{p}_{I_{j}}\right)$ and ${ }^{I} \boldsymbol{\ell}_{j}$ to obtain the covariance of the initial position estimate. However, similar to Section IV-B.2, the estimates of the IMU pose at different time instants are correlated; thus, we again employ stochastic cloning [27] to account for their correlations when computing the covariance of the initial position estimate, ${ }^{G} \hat{\mathbf{p}}_{I}$.

\section{OBSERVABILITy ANALYSis}

In this section, we prove that the presented system for IMU-laser scanner localization is observable when three planes, whose normal vectors are linearly independent, are concurrently observed by the laser scanner. Under this condition, and since the IMU and the laser scanner are rigidly connected and their relative transformation is known, we can employ the pose estimation method described in Section IV to estimate $\left({ }^{G} \mathbf{p}_{I},{ }^{I} \bar{q}_{G}\right)$. For the purpose of observability analysis, we introduce two new inferred measurements ${ }^{4} \mathbf{h}_{1}^{*}$ and $\mathbf{h}_{2}^{*}$ that replace the laser scan measurements (17), (21):

$$
\begin{aligned}
{ }^{I} \bar{q}_{G} & =\mathbf{h}_{1}^{*}(\mathbf{x})=\boldsymbol{\xi}_{1}\left({ }^{I} \boldsymbol{\ell}_{1},{ }^{I} \boldsymbol{\ell}_{2},{ }^{I} \boldsymbol{\ell}_{3}\right) \\
{ }^{G} \mathbf{p}_{I} & =\mathbf{h}_{2}^{*}(\mathbf{x})=\boldsymbol{\xi}_{2}\left({ }^{I} \boldsymbol{\ell}_{1},{ }^{I} \boldsymbol{\ell}_{2},{ }^{I} \boldsymbol{\ell}_{3}\right) .
\end{aligned}
$$

The two functions $\boldsymbol{\xi}_{1}$ and $\boldsymbol{\xi}_{2}$ in (31) and (32) do not need to be known explicitly; only their functional relation with the random variables, ${ }^{I} \bar{q}_{G}$ and ${ }^{G} \mathbf{p}_{I}$, is required for the observability analysis. Our approach uses the Lie derivatives [4] of the above inferred measurements (31) and (32) for the system in (2)-(4), to show that the corresponding observability matrix is full rank. For this purpose, we first rearrange the nonlinear kinematic equations (2)-(4) in a suitable form for computing the Lie derivatives:

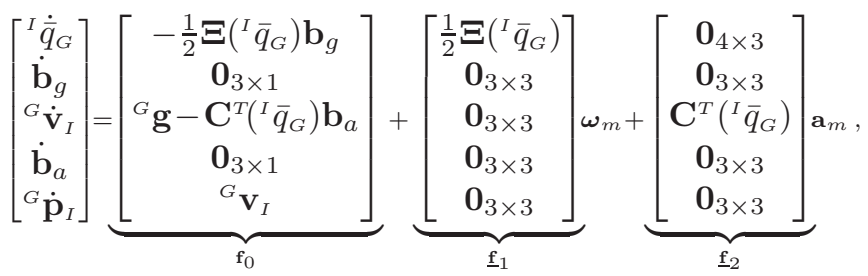

\footnotetext{
${ }^{4}$ The observability of the system can also be shown using only the inferred position measurement (32). For details refer to [28].
} 


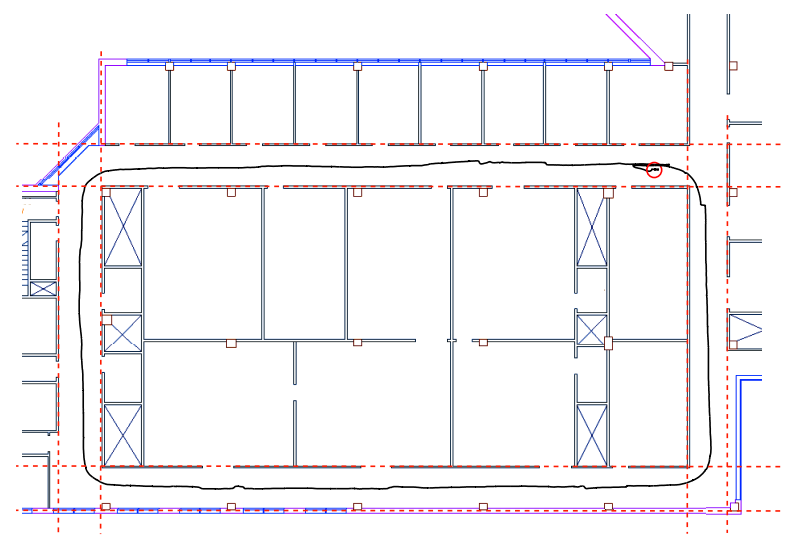

Fig. 2. Top-view of the estimated 3D trajectory during an $8.5 \mathrm{~min}$ experiment. The total length of the trajectory is $120 \mathrm{~m}$. The red circle indicates the starting position (on the floor), then the sensor package was picked up and traced a clock-wise loop through the building hallways. The dashed red lines indicate the walls which were included in the building map.

where $\boldsymbol{\omega}_{m}$ and $\mathbf{a}_{m}$ are considered the control inputs, and

$$
\boldsymbol{\Xi}(\bar{q}) \triangleq\left[\begin{array}{c}
q_{4} \mathbf{I}_{3}+\lfloor\mathbf{q} \times\rfloor \\
-\mathbf{q}^{T}
\end{array}\right] \quad \text { with } \quad \bar{q}=\left[\begin{array}{c}
\mathbf{q} \\
q_{4}
\end{array}\right] .
$$

Note also that $\mathbf{f}_{0}$ is a $16 \times 1$ vector, while $\underline{\mathbf{f}}_{1}$ and $\underline{\mathbf{f}}_{2}$ are matrices of dimensions $16 \times 3$.

In order to prove that the system is locally weakly observable, it suffices to show that the observability matrix, whose rows comprise the gradients of the Lie derivatives of the measurements $\mathbf{h}_{1}^{*}$ and $\mathbf{h}_{2}^{*}$ with respect to $\mathbf{f}_{0}, \underline{\mathbf{f}}_{1}$, and $\underline{\mathbf{f}}_{2}$ [cf. (33)], is full rank. Since the measurement and kinematic equations describing the IMU-laser scanner localization are infinitely smooth, the observability matrix has an infinite number of rows. However, to prove it is full rank, it suffices to show that a subset of its rows are linearly independent. The following matrix contains one such subset of rows whose linear independence can be easily shown using block Gaussian elimination [28]:

$\left[\begin{array}{c}\nabla \mathfrak{L}_{\mathfrak{f}_{0}^{0}}^{0} \mathbf{h}_{1}^{*} \\ \nabla \mathfrak{L}_{\mathfrak{f}_{0}^{0}}^{0} \mathbf{h}_{2}^{*} \\ \nabla \mathfrak{L}_{\mathfrak{f}^{1}}^{1} \mathbf{h}_{1}^{*} \\ \nabla \mathfrak{L}_{\mathfrak{f}_{0}^{1}}^{\mathfrak{f}_{0}} \mathbf{h}_{2}^{*} \\ \nabla \mathfrak{L}_{\mathbf{f}_{0}}^{2} \mathbf{h}_{2}^{*}\end{array}\right]=\left[\begin{array}{ccccc}\mathbf{I}_{4} & \mathbf{0}_{4 \times 3} & \mathbf{0}_{4 \times 3} & \mathbf{0}_{4 \times 3} & \mathbf{0}_{4 \times 3} \\ \mathbf{0}_{3 \times 4} & \mathbf{0}_{3 \times 3} & \mathbf{0}_{3 \times 3} & \mathbf{0}_{3 \times 3} & \mathbf{I}_{3} \\ \mathbf{X}_{1} & \left.-\frac{1}{2} \mathbf{\Xi}^{I} \bar{q}_{G}\right) & \mathbf{0}_{4 \times 3} & \mathbf{0}_{4 \times 3} & \mathbf{0}_{4 \times 3} \\ \mathbf{0}_{3 \times 4} & \mathbf{0}_{3 \times 3} & \mathbf{I}_{3} & \mathbf{0}_{3 \times 3} & \mathbf{0}_{3 \times 3} \\ \mathbf{X}_{2} & \mathbf{0}_{3 \times 3} & \mathbf{0}_{3 \times 3} & \mathbf{C}^{T}\left({ }^{I} \bar{q}_{G}\right) & \mathbf{0}_{3 \times 3}\end{array}\right]$

where $\mathfrak{L}_{\mathbf{f}_{0}}^{i} \mathbf{h}_{j}^{*}(\mathbf{x})$ denotes the $i$-th order Lie derivative of $\mathbf{h}_{j}^{*}(\mathbf{x})$ with respect to $\mathbf{f}_{0}$. The matrices $\mathbf{X}_{1}$ and $\mathbf{X}_{2}$ have dimensions $4 \times 4$ and $3 \times 4$, respectively, and do not need to be computed explicitly since they will be eliminated by the block element $(1,1)$ of the matrix, i.e., the identity matrix $\mathbf{I}_{4}$. Since $\boldsymbol{\Xi}(\bar{q})$ and $\mathbf{C}(\bar{q})$ are always full rank for any unit quaternion $\bar{q}$ [28], all the rows of the above matrix are linearly independent. Hence, we conclude the observability analysis with the following lemma:

Lemma 1: Given line measurements corresponding to three planes with linearly independent normal vectors, the system describing the IMU-laser scanner localization is locally weakly observable.

\section{EXPERIMENTAL RESULTS}

The 6 d.o.f. IMU-laser localization algorithm was tested in an indoor environment, along a closed-loop trajectory of approximately $120 \mathrm{~m}$ in length (cf. Fig. 2). We utilized a solid-state ISIS IMU and a SICK LMS200 laser scanner mounted on a navigation box to log data. These sensors were interfaced to a laptop via RS-232 which recorded the time-stamped measurements. The data-logging software was implemented in $\mathrm{C}++$, whereas the EKF was written in MATLAB.

During the experiment ${ }^{5}$, the motion profile of the sensor platform contained instantaneous stationary time periods to allow for zero-velocity updates. These updates cause small reductions in the position estimates' covariance [cf. Fig. 3(a)]. Larger reductions in the covariance take place whenever the laser scanner detects three planes whose normal vectors are linearly independent (e.g., two perpendicular walls and the ceiling) within a short period of time; an event that typically occurs at hallway intersections (e.g., $t=49 \mathrm{sec}$ ). The a priori known map, available from the building blueprints, contained 9 walls and the ceiling. Employing this map, nearly 12,000 measurement updates were performed during the 8.5 minute trial. The combination of the laser measurements and zero-velocity updates allowed the filter to maintain a precise pose estimate of the sensor platform. Specifically, the maximum uncertainty in the position estimates was $9.16 \mathrm{~cm}(1 \sigma)$, while the maximum uncertainty in the attitude estimates was $0.1 \mathrm{deg}$ $(1 \sigma)$ [cf. Figs. 3(a), 3(b)]. The final position uncertainty was $\left[\begin{array}{lll}27.5 & 1.2 & 1.3\end{array}\right] \mathrm{cm}(3 \sigma)$. Note that the $x$-direction uncertainty is larger since in the final corridor, no planes are observed that provide information along the $x$-axis. Finally, the filter consistency is corroborated by the measurement residuals which lie within their $3 \sigma$ bounds [cf. Fig. 3(c)].

\section{CONClusions AND Future Work}

This paper presented a novel approach to 3D map-based indoor localization for the visually impaired using information from an IMU and a 2D laser scanner. Linear accelerations and rotational velocities are integrated to propagate the pose estimates. To update the pose, measurements from the laser scanner are used, which correspond to the intersection of the laser-scan plane with known structural planes of an indoor environment. We addressed several challenges including: (i) solving the 6 d.o.f. pose estimation problem using an IMU and 2D laser scanner, (ii) determining a sufficient condition for the system observability, and (iii) initializing the pose estimate and covariance in a practical way. We also demonstrated the validity of our algorithm with experimental results showing that we can provide reliable pose estimates of the person.

Our future work includes employing small-scale sensors to enable a hand-held sensor package. Providing an efficient and intuitive system interface for a visually impaired person, is also within our near goals. Finally, we intend to extend

\footnotetext{
${ }^{5}$ Video available at http://mars.cs.umn.edu/videos/IMU-Laser.m4v
} 


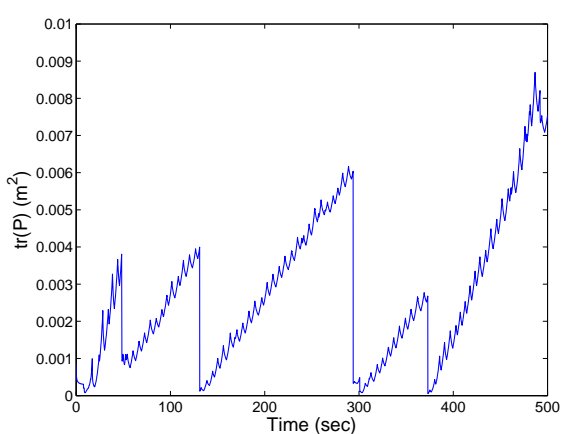

(a)

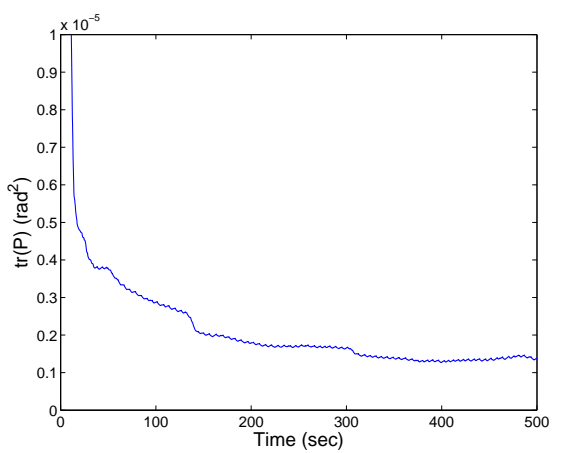

(b)
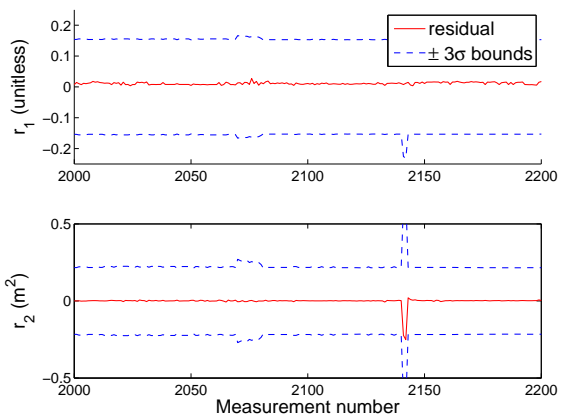

(c)

Fig. 3. (a) The trace of the position covariance. During the run, the maximum uncertainty along any axis was $9.16 \mathrm{~cm}$. ( $1 \sigma$ ). (b) The trace of the attitude covariance. During the run, the maximum uncertainty about any axis was $0.1 \mathrm{deg}$. $(1 \sigma)$. (c) More than 12,000 line measurements were recorded during this experiment. The measurement residuals for a subset of these (200) are plotted in order to demonstrate the filter consistency.

our work to cases when building blueprints are not available.

\section{REFERENCES}

[1] S. Shoval, J. Borenstein, and Y. Koren, "Auditory guidance with the NavBelt - a computerized travel aid for the blind," IEEE Trans. on Systems, Man, and Cybernetics, vol. 28, no. 3, pp. 459-467, Aug. 1998.

[2] J. A. Hesch and S. I. Roumeliotis, "An indoor localization aid for the visually impaired," in Proc. of the IEEE Int. Conf. on Robotics and Automation, Rome, Italy, Apr. 10-14, 2007, pp. 3545-3551.

[3] H. H. Chen, "Pose determination from line-to-plane correspondences: existence condition and closed-form solutions," IEEE Trans. on Pattern Analysis and Machine Intelligence, vol. 13, no. 6, pp. 530-541, June 1991.

[4] R. Hermann and A. Krener, "Nonlinear controlability and observability," IEEE Trans. on Automatic Control, vol. 22, no. 5, pp. 728-740, Oct. 1977.

[5] D. Yuan and R. Manduchi, "Dynamic environment exploration using a virtual white cane," in Proc. of the IEEE Conf. on Computer Vision and Pattern Recognition, San Diego, CA, June 20-25, 2005, pp. 243249.

[6] D. A. Bell, J. Borenstein, S. P. Levine, Y. Koren, and L. Jaros, "An assistive navigation system for wheelchairs based on mobile robot obstacle avoidance," in Proc. of the IEEE Int. Conf. on Robotics and Automation, San Diego, CA, May 8-13, 1994, pp. 2018-2022.

[7] I. Ulrich and J. Borenstein, "The GuideCane - applying mobile robot technologies to assist the visually impaired," IEEE Trans. on Systems, Man, and Cybernetics, Part A: Systems and Humans, vol. 31, no. 2, pp. 131-136, Mar. 2001.

[8] A. Hub, J. Diepstraten, and T. Ertl, "Design and development of an indoor navigation and object identification system for the blind," in Proc. of the Int. ACM SIGACCESS Conf. on Computers and Accessibility, Atlanta, GA, Oct. 18-20, 2004, pp. 147-152.

[9] H. Makino, I. Ishii, and M. Nakashizuka, "Development of navigation system for the blind using GPS and mobile phone combination," in Proc. of the IEEE Int. Conf. of the Engineering in Medicine and Biology Society, Amsterdam, Netherlands, Oct. 31-Nov. 3, 1996, pp. 506-507.

[10] L. Ran, S. Helal, and S. Moore, "Drishti: an integrated indoor/outdoor blind navigation system and service," in Proc. of the IEEE Conf. on Pervasive Computing and Communications, Orlando, FL, Mar. 14-17, 2004, pp. 23-30.

[11] F. Cavallo, A. M. Sabatini, and V. Genovese, "A step toward GPS/INS personal navigation systems: real-time assessment of gait by foot inertial sensing," in Proc. of the IEEE/RSJ Int. Conf. on Intelligent Robots and Systems, Edmonton, Canada, Aug. 2-6, 2005, pp. 11871191.

[12] K. Sagawa, H. Inooka, and Y. Satoh, "Non-restricted measurement of walking distance," in Proc. of the IEEE Int. Conf. on Systems, Man, and Cybernetics, Nashville, TN, Oct. 8-11, 2000, pp. 1847-1852.

[13] V. Kulyukin, C. Gharpure, J. Nicholson, and S. Pavithran, "RFID in robot-assisted indoor navigation for the visually impaired," in Proc. of the IEEE/RSJ Int. Conf. on Intelligent Robots and Systems, Sendai, Japan, Sept. 28-Oct. 2, 2004, pp. 1979-1984.
[14] B. Tjan, P. Beckmann, N. Giudice, and G. Legge, "Digital sign system for indoor wayfinding for the visually impaired," in Proc. of the IEEE Conf. on Computer Vision and Pattern Recognition - Workshop on Computer Vision Applications for the Visually Impaired, San Diego, CA, June 20-25, 2005.

[15] S. Ertan, C. Lee, A. Willets, H. Tan, and A. Pentland, "A wearable haptic navigation guidance system," in Proc. of the Int. Sym. on Wearable Computers, Pittsburgh, PA, Oct. 19-20, 1998, pp. 164-165.

[16] F. Dellaert and S. Tariq, "A multi-camera pose tracker for assisting the visually impaired," in Proc. of the IEEE Conf. on Computer Vision and Pattern Recognition - Workshop on Computer Vision Applications for the Visually Impaired, San Diego, CA, June 20-25, 2005.

[17] O. Koch and S. Teller, "Wide-area egomotion estimation from known 3D structure," in Proc. of the IEEE Conf. on Computer Vision and Pattern Recognition, Minneapolis, MN, June 17-22, 2007, pp. 1-8.

[18] W. Rungsarityotin and T. E. Starner, "Finding location using omnidirectional video on a wearable computing platform," in Proc. of the IEEE Int. Symposium on Wearable Computing, Washington, DC, 2000, pp. 61-68.

[19] G. Weiß, C. Wetzler, and E. V. Puttkamer, "Keeping track of position and orientation of moving indoor systems by correlation of rangefinder scans," in Proc. of the IEEE/RSJ Int. Conf. on Intelligent Robots and Systems, Munich, Germany, Sept. 12-16, 1994, pp. 595-601.

[20] F. Lu and E. Milios, "Robot pose estimation in unknown environments by matching 2D range scans," Journal of Intelligent and Robotic Systems, vol. 18, pp. 249-275, Mar. 1997.

[21] K. Lingemann, A. Nuchter, J. Hertzberg, and H. Surmann, "Highspeed laser localization for mobile robots," Robotics and Autonomous Systems, vol. 51, no. 4, pp. 275-296, Apr. 2005.

[22] E. J. Lefferts, F. L. Markley, and M. D. Shuster, "Kalman filtering for spacecraft attitude estimation," Journal of Guidance, Control, and Dynamics, vol. 5, no. 5, pp. 417-429, Sept.-Oct. 1982.

[23] N. Trawny and S. I. Roumeliotis, "Indirect Kalman filter for 3D pose estimation," MARS Lab, Dept. of Computer Science \& Engineering, University of Minnesota, Minneapolis, MN, Tech. Rep., Mar. 2005.

[24] V. Nguyen, S. Gächter, A. Martinelli, N. Tomatis, and R. Siegwart, "A Comparison of Line Extraction Algorithms using 2D Range Data for Indoor Mobile Robotics," Autonomous Robots, vol. 23, no. 2, pp. 97-111, Aug. 2007.

[25] S. T. Pfister, S. I. Roumeliotis, and J. W. Burdick, "Weighted line fitting algorithms for mobile robot map building and efficient data representation," in Proc. of the IEEE Int. Conf. on Robotics and Automation, Sept. 14-19, 2003, pp. 1304-1311.

[26] F. M. Mirzaei and S. I. Roumeliotis, "A kalman filter-based algorithm for IMU-camera calibration: Observability analysis and performance evaluation," IEEE Trans. on Robotics, vol. 24, no. 5, pp. 1143-1156, Oct. 2008.

[27] A. I. Mourikis, S. I. Roumeliotis, and J. W. Burdick, "SC-KF mobile robot localization: A stochastic cloning kalman filter for processing relative-state measurements," IEEE Trans. on Robotics, vol. 23, no. 4, pp. 717-730, Aug. 2007.

[28] F. M. Mirzaei and S. I. Roumeliotis, "IMU-laser scanner localization: Observability analysis," Dept. of Computer Science \& Engineering, University of Minnesota, MARS Lab, Minneapolis, MN, Tech. Rep., Jan. 2009. [Online]. Available: http://mars.cs.umn.edu/tr/reports/Mirzaei09.pdf 